\title{
Focused-probe STEM Ptychography: Developments and Opportunities
}

Colum O’Leary ${ }^{1}$, Emanuela Liberti ${ }^{1}$, Gerardo Martinez ${ }^{1}$, Christopher Allen ${ }^{2}$, Chen Huang ${ }^{1}$, Mathias Rothmann ${ }^{1}$, Hui Luo ${ }^{1}$, Judy Kim ${ }^{2}$, Laura Herz ${ }^{1}$, Hazel Assender ${ }^{1}$, Lewys Jones ${ }^{3}$, Angus Kirkland ${ }^{1}$ and Peter Nellist ${ }^{1}$

${ }^{1}$ University of Oxford, Oxford, England, United Kingdom, ${ }^{2}$ Electron Physical Science Imaging Centre, Diamond Light Source, Didcot, England, United Kingdom, ${ }^{3}$ Trinity College Dublin, Dublin, Dublin, Ireland

The advent of fast pixelated detectors for electron microscopy has given rise to an explosion of fourdimensional scanning transmission electron microscopy (4D STEM) applications over the last decade [1], not least in the field of focused-probe STEM ptychography (FPSP) [2]. FPSP utilizes the interference in convergent beam electron diffraction (CBED) patterns to detect the sample-induced phase shifts of the electron wave. With super-resolution capabilities, post-processing aberration correction and efficient phase contrast imaging versus other STEM imaging techniques [3], FPSP has significant potential for atomic resolution imaging of light elements and beam-sensitive materials. However, slow scanning speeds $(1 \mathrm{kHz})$, narrow information transfer and high standard beam currents $(>1 \mathrm{pA})$ has rendered FPSP challenging to achieve on such materials.

This presentation will explore the experimental and analytical approaches to minimizing electron dose (electrons per unit area) and maximizing dose efficiency of FPSP. Various direct electron detectors enable acquisition speed to be increased via binning (up to $7.5 \mathrm{kHz}$ [4] in integrating mode); others do so by adjusting their dynamic range (up to $12.5 \mathrm{kHz}$ [5] in counting mode). Using such methods has enabled phase reconstructions of zeolites at cumulative electron doses below $1000 \mathrm{e} \AA^{-2}$, as shown in Figure 1 . The choice of convergence angle and sampling settings can be optimized before data acquisition to minimize the cumulative electron dose. When the data is dominated by noise, 4D STEM acquisition can be performed using multiple fast scans before performing non-rigid registration and averaging, mitigating scan distortions and sample drift which can be present in a single, slower scan [6]. In addition to optimizing experimental parameters, post-processing techniques can be applied to maximize the dose efficiency of the reconstruction. For example, aberration correction can be performed in post-processing, thus removing the need to expose the sample to the beam before data acquisition. Furthermore, noise normalization can increase the window of information transfer in phase reconstructions, such that both low and high spatial frequencies are transferred with high contrast. These approaches will be described together with their application to a range of beam-sensitive samples, such as zeolites and crystalline polymers. The commercialization of prototype fast pixelated detectors with frame rates close to $0.1 \mathrm{MHz}$ is in future expected to play a major role in further enabling low dose FPSP experiments [7],[8]. 

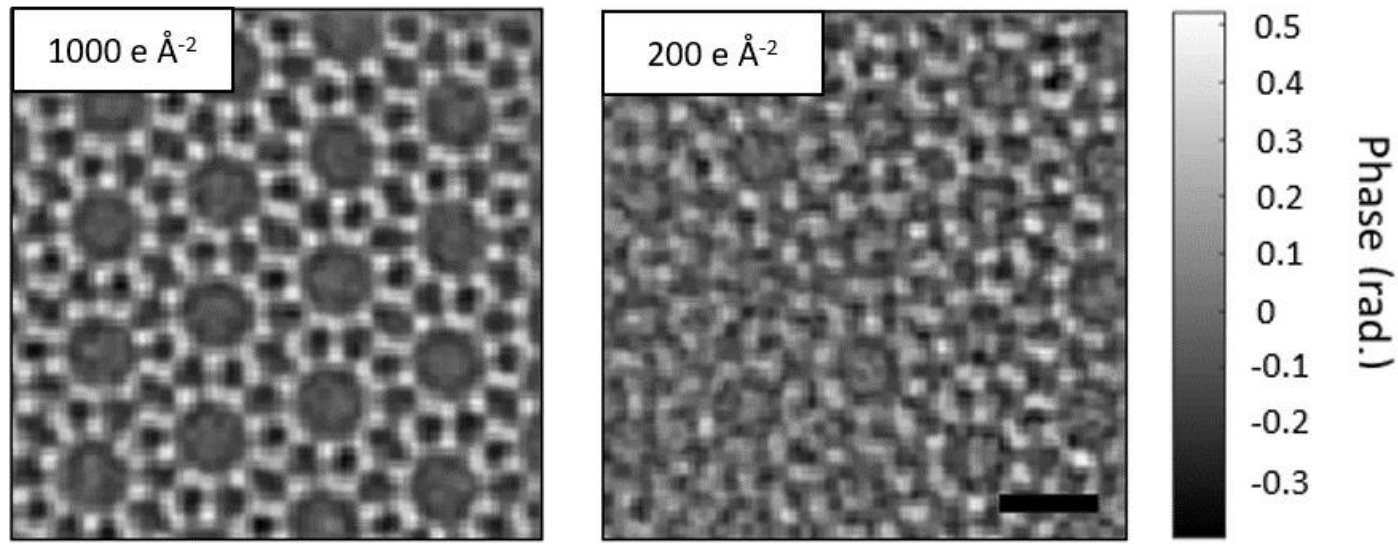

Figure 1. Ptychographic phase reconstruction of ZSM-5 zeolite using a MerlinEM fast pixelated detector with 1-bit counting depth at a frame rate of $12.5 \mathrm{kHz}$. Scale bar: $1 \mathrm{~nm}$. Adapted from Appl. Phys. Lett. 116, 124101 (2020), with the permission of AIP Publishing. DOI: https://doi.org/10.1063/1.5143213.

\section{References}

[1] C. Ophus, Microscopy and Microanalysis, vol. 25, no. 3, pp. 563-582, 2019.

[2] J. G. Lozano et al., Nano Lett., vol. 18, no. 11, pp. 6850-6855, 2018.

[3] T. J. Pennycook et al., Ultramicroscopy, vol. 151, pp. 160-167, 2015.

[4] M. Huth et al., Microscopy and Microanalysis, vol. 25, no. S2, pp. 40-41, 2019.

[5] C. M O'Leary et al., Microscopy and Microanalysis vol. 25, no. S2, pp. 1662-1663, 2019.

[6] L. Jones et al., Advanced Structural and Chemical Imaging, vol. 1, no. 8, 2015.

[7] J. Ciston et al., Microscopy and Microanalysis, vol. 25, no. S2, pp. 1930-1931, 2019.

[8] The financial support of the EPSRC, The Henry Royce Institute and JEOL (UK) Ltd. Is gratefully acknowledged. We thank Diamond Light Source for access and support in use of the electron Physical Sciences Imaging Centre (Instrument E02, proposal numbers MG20431 and MG22317) that contributed to the results presented here. 